\title{
Die Leiden des Niccolo Paganini
}

\author{
The Diseases of Niccolo Paganini
}

\section{Bibliografie}

DOI $10.1055 / \mathrm{s}-0028-1119679$

Online-Publikation: 16.3.2009

Akt Dermatol 2009; 35:

411-414 @ Georg Thieme

Verlag KG Stuttgart · New York ISSN 0340-2541

Korrespondenzadresse Dr. Manfred Reitz

Walther-Victor-Straße 20 99425 Weimar

reitzmanfred@yahoo.de

\section{Zusammenfassung \\ $\nabla$}

Der italienische Geiger und Komponist Niccolo Paganini wurde zu seinen Lebzeiten in ganz Europa wegen seiner virtuosen Spielweise als Wundergeiger verehrt. Er erfand Geigengriffe, die kein anderer Geiger nachvollziehen konnte. Er war in der Lage, seine Gelenke zu überdehnen und zu überdrehen. Heute wird angenommen, dass Paganini ein einem Marfan-Syndrom erkrankt ge-

\section{Einleitung \\ $\nabla$}

Im frühen 19. Jahrhundert erreichte der italienische Geiger und Komponist Niccolo Paganini (1782 - 1840) bei seinem Publikum einen Status, wie er heute höchstens einem Popstar zukommt. Die Besucher seiner Konzerte vergötterten ihn, und er war für sie ein Wundergeiger. Paganini gilt bis heute als das Urbild eines Geigenvirtuosen, der durch seine unglaubliche Spieltechnik regelmäßig Begeisterungsstürme auslösen kann. In Wien erhielt er 1828 sogar den Ehrentitel eines kaiserlichen Kammervirtuosen. Für sein Publikum stand er mit dem Teufel im Bunde. Er beherrschte in seinem Spiel Geigengriffe, die keiner seiner Konkurrenten nachvollziehen konnte. Insbesondere die Damen der feinen Salons bewunderten seine einmalige Virtuosität und priesen ihn als Meister und Dämon. Andere Geiger wurden neidisch auf ihn. Trotz aller Übung gelang es ihnen nicht, die Spieltechnik des Niccolo Paganini wirklich exakt zu kopieren. Sie scheiterten meist an seinen äußerst schwierigen Geigengriffen. Eine seiner Spezialitäten war die perfekte Übereinstimmung zwischen einem gleichzeitigen wilden Bogenstrich mit der rechten Hand und einem schwierigen Pizzikato mit der linken Hand; dem vom Bogenstrich unabhängigen Zupfen der Saiten mit den Fingern. Gestrichene Melodien wurden von ihm sogar mehrstimmig mit einem Pizzikato begleitet. wesen war; ein Erbdefekt, den die Medizin zu seinen Lebzeiten noch nicht kannte. Erst durch seine Erkrankung konnte Paganini zu einem unerreichbaren Geigenvirtuosen werden. Bei den heutigen Nachkommen von Paganini gibt es jedoch keine Diagnosen eines Marfan-Syndroms. Defekte Fibrillin-Gene wurden bei ihnen nicht nachgewiesen. Von Paganini selbst ist keine DNA-Probe erhalten.

Nach Meinung seines Publikums konnte Paganini während seiner Konzerte gleichzeitig Trauer, Sehnsucht und Leidenschaft in sein Spiel einfließen lassen und dadurch jeden Konzertbesucher in seinen Gefühlen packen. Von Paganini angeregt, entwickelte Franz List einen hochvirtuosen Klavierstil und wurde anschließend ähnlich seinem Vorbild ebenfalls populär. Für Musiker, die technisch außergewöhnlich gut sind, schrieb der Komponist Schumann seine „Paganini-Etüden“ (1833) und der Komponist Brahms seine „Paganini-Variationen“ (1863).

Die Begeisterung seines Publikums machte Paganini zu einem reichen Mann. Im Jahre 1831 brachte ihm beispielsweise eine Konzertreise durch Frankreich innerhalb von nur drei Monaten einen Gewinn von rund 153000 Francs ein. Auf seinen europaweiten Konzertreisen verlangte er meist weit überhöhte Eintrittspreise, dennoch drängte sich das Publikum. An einen Freund und finanziellen Berater in Genua schrieb er einmal: „Paris, London und Russland werden meine Million vervollständigen.“ Er konnte sich deshalb auch erlauben, Konzerte für mildtätige Zwecke zu geben und die Einnahmen zu spenden. Dem französischen Komponisten Hector Belioz schenkte er einmal 20000 Francs. Vom heutigen Standpunkt aus gesehen war Paganini ein vielfacher Millionär. In seinem Nachlass fanden sich 
neben anderen wertvollen Musikinstrumenten auch sieben Geigen von Stradivari und vier von Guarneri [1,2].

\section{Die Krankheiten des Niccolo Paganini}

Von Paganini sind zahlreiche Abbildungen erhalten, die ihn bei seinen Konzerten zeigen ( $\bullet$ Abb. $\mathbf{1})$.

Bei einer Zeichnung von Ch. Sawyer (1831) fällt beispielsweise sofort eine recht merkwürdige Körperhaltung auf. Während sich andere Geiger stets artig in streng aufrechter und gerader Haltung vor dem Publikum präsentierten, spreizte Paganini beim Spielen ein Bein ab und hielt den Oberkörper mitsamt den Armen seltsam überdreht. Einem normalen Menschen würde es schwerfallen, eine solche Körperhaltung einzunehmen und gleichzeitig noch für eine längere Zeit durchzuhalten. In Karikaturen wurde Paganini wegen dieser unkonventionellen Körperhaltung, seiner oft wirren schwarzen Haare und seiner schwarzen Konzertkleidung gern als eine Art von Hexenmeister dargestellt, den die Damen anhimmelten.

In seinen letzten Lebensjahren war Paganini ein schwer kranker Mann, denn es summierten sich immer mehr die Folgen von Erkrankungen aus vergangenen Lebensabschnitten. Nach seinem Tod am 27. Mai 1840 veröffentliche sein Arzt und Freund Francesco Bennati erst 1845 ein Gutachten über die Erkrankungen des großen Meisters. In jungen Jahren war Paganini an einer Masernenzephalitis erkrankt gewesen, an deren Folgen er für den Rest seines Lebens litt. In seinen mittleren Jahren erfolgten zusätzlich noch eine Infektion an Syphilis sowie an Tuberkulose, die beide seinen Gesundheitszustand ständig verschlechterten. Im Alter schließlich stellte sich eine Kehlkopftuberkulose mit einem Verlust der Stimme sowie eine Knochennekrose des Unterkiefers ein, er verlor nun nach und nach seine Zähne. Die direkte Todesursache war zuletzt nach einem längeren Krankenlager in Nizza ein Blutsturz. Bennati berichtete auch über vielfältige Beschwerden in den Funktionen der Harnblase, der Prostata und des Dickdarms. Weitere Hinweise liegen ebenfalls über seine immer wiederkehrende Hautschädigungen vor. Sie sollen für sein manchmal dämonisches und gespenstisches Aussehen verantwortlich gewesen sein.

Für den schlechten Gesundheitszustand von Paganini waren sicherlich auch die zahlreichen Medikamente verantwortlich, die er zur Behandlung seiner Leiden über viele Jahre einnehmen musste. Im frühen 19. Jahrhundert wurden noch viele Medikamente gegen Infektionen auf der Grundlage von Quecksilber hergestellt, sodass bei Patienten zahlreiche schädliche Nebenwirkungen zu erwarten waren. Manche Beschwerden von Paganini gingen sicherlich auf erhöhte Quecksilberkonzentrationen in seinem Organismus zurück. Vor einigen Jahren wurden von ihm einige erhaltene und nachweislich echte Haarproben untersucht und Schadstoffkonzentrationen analysiert. Es konnte eine erschreckend hohe Konzentration von Quecksilber nachgewiesen werden [3].

\section{Das Bild des Niccolo Paganini \\ $\nabla$}

Bennati ging in seinem Krankheitsbericht auch auf die äußere Erscheinung von Paganini ein. Er beschrieb dessen hohen Körperwuchs, die schlanke Gestalt und das spinnenartige Aussehen der Hände. Ihm war ebenfalls aufgefallen, dass der Meister in der Lage war, seine Finger zu überdehnen und er nach seiner ärztli-

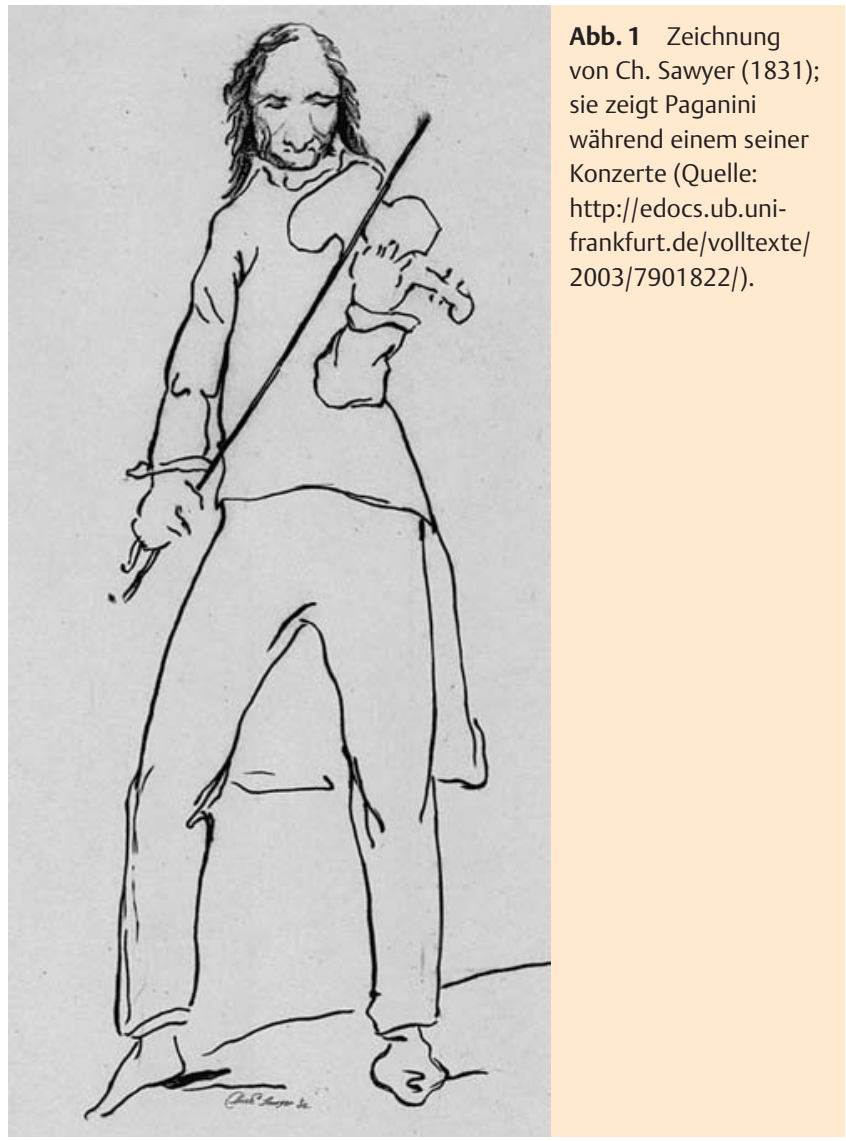

chen Meinung nur durch diese Fähigkeit seine Virtuosität erreichen konnte. Lange nach dem Tod von Niccolo Paganini äußerten Ärzte nach der Lektüre dieses Berichts erstmals die Meinung, dass der Meister eventuell an einem Marfan-Syndrom erkrankt gewesen sein könnte. Zu Lebzeiten von Paganini war diese Erkrankung mit ihren komplexen Symptomen noch nicht beschrieben worden und sie ist auch heute noch nicht immer einfach zu diagnostizieren. Unter den Vorfahren von Paganini gibt es keine Hinweise auf ein Marfan-Syndrom. Allerdings ist zu bedenken, dass der Erbdefekt Marfan-Syndrom in etwa einem Viertel der Fälle auf eine Neumutation zurückgeht und nicht von einem Elternteil stammt.

Erhalten ist der Gipsabdruck einer Hand von Paganini, der nach seinem Tod angefertigt wurde.

Fachleute erkennen in dieser Hand die langen dünnen und als spinnenartig beschriebenen Finger, die für Patienten mit Marfan-Syndrom typisch sind [3].

\section{Die Beobachtungen des Professor Dequeker}

Jan Dequeker ist Professor für Rheumatologie an der Universität von Leuven in Belgien. Er gilt als ein hervorragender Fachmann für Diagnosen und nutzt seine Fähigkeiten zusätzlich noch für ein ausgefallenes Hobby: Dequeker ist ein Freund der Kunst und besucht regelmäßig Ausstellungen mit einem Blick für ärztliche Diagnosen. Wie andere Kunstfreunde ist zwar auch er vom künstlerischen Wert von Porträts oder Figurendarstellungen begeistert, doch noch mehr interessieren ihn bei den dargestellten Menschen Erkrankungen, deren Symptome von den Künstlern, ohne es vermutlich bewusst zu merken, abgebildet wurden. Bei 


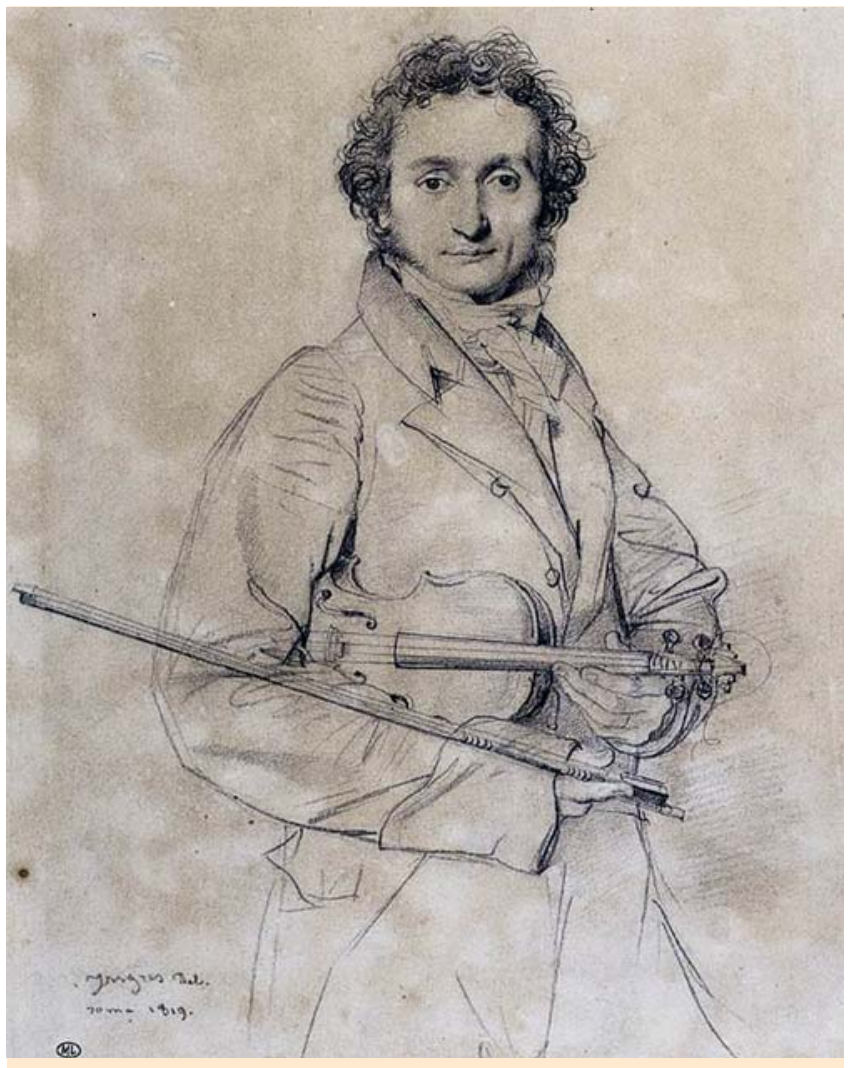

Abb. 2 Zeichnung von Jean-Auguste Ingres (1819); das Porträt stellt Paganini dar (Quelle: Wikipedia).

einem Bild von Paganini ( $\bullet$ Abb. 2), das der französische Maler Ingres 1819 anfertigte, war sich Dequeker spontan sicher, dass der Wundergeiger an einem Marfan-Syndrom erkrankt gewesen sein musste. Dabei beurteilte er insbesondere das Aussehen des Gesichts und die Finger des Meisters.

Auf dem Gemälde „Die drei Grazien“ im Madrider Prado stellte Rubens seine zweite Ehefrau Hélène Fourment und zwei ihrer Schwestern als Akte dar ( Abb. 3).

Rubens malte seine Modelle sehr präzise und wäre heute sicherlich überrascht, dass Dequeker bei ihnen gleich eine Liste von Erkrankungen und sogar Schwächen in den Bindegeweben diagnostizieren konnte. Bereits dem Laien fällt auf, dass die Grazie in der Mitte des Bildes an einer Skoliose leidet. Doch Dequeker erkennt viel mehr: Die mittlere Grazie steht auf dem linken Bein, trotzdem ist die rechte Hüfte herabgesunken. Dequeker sieht darin ein „positives Trendelburg-Zeichen“. Das Becken kann nicht im Gleichgewicht gehalten werden, was sich eventuell auf eine Hüftluxation oder eine Insuffizienz der Gesäßmuskeln zurückführen lässt. Die Grazie auf der linken Seite besitzt nach der Meinung von Dequeker Plattfüße und leidet aufgrund von bestimmten Fingerstellungen an Arthritis. Bei allen drei Frauen glaubt Dequeker eine Hyperlordose in der Lendenwirbelsäule zu erkennen. Außerdem zeichnen sich nach seiner Meinung alle drei Frauen durch ein familiäres Hypermobilitätssyndrom aus, eine Bindegewebsschwäche, die eine Überbeweglichkeit von Gelenken zur Folge hat.

Die drei Gazien waren nach den Mythen Töchter des Göttervaters Zeus und Gespielinnen der Liebesgöttin Aphrodite. Heute wird sich Zeus auf dem Olymp wundern, wie krank seine hübschen Töchter waren $[4,5]$.

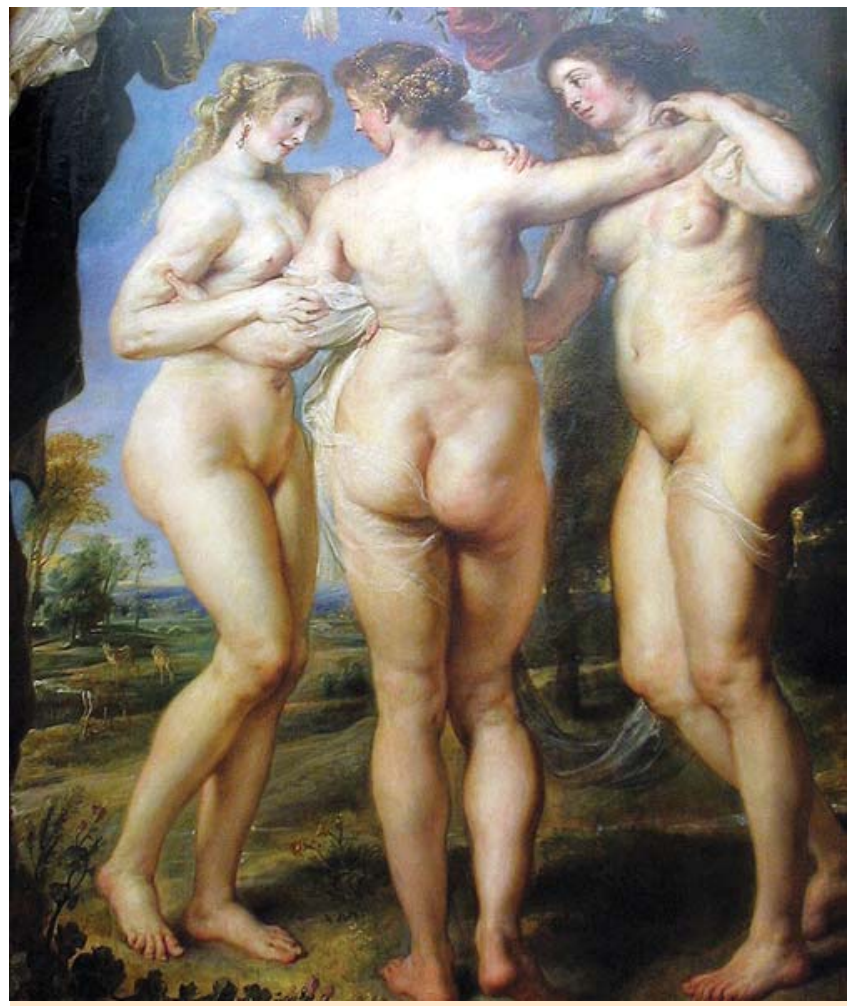

Abb. 3 „Die drei Grazien“ von Peter Paul Rubens; Jan Dequeker diagnostizierte bei den Modellen verschiedene Erkrankungen (Quelle: Wikipedia).

\section{Das Marfan-Syndrom}

Das Marfan-Syndrom gehört zu den angeborenen Störungen des Bindegewebes und ist somit genetisch bedingt. Es geht auf eine Mutation im Gen zur Synthese von Fibrillin zurück und wird autosomal dominant vererbt. Fibrillin ist ein Bestandteil von elastischen Fasern in den Mikrofibrillen der Bindegewebe. Bei Fehlbildungen vermindert sich ihre Resistenz gegenüber mechanischen Belastungen. Kompliziert wird die Angelegenheit, weil das Gen für Fibrillin gleich auf zwei Chromosomen lokalisiert ist; auf Chromosom 15q21 kommt das Fibrillin-Gen FBN1 vor und auf Chromosom 5q21 das Fibrillin-Gen FBN2. Bei Mutationen im Fibrillin-Gen FBN2 sind die Erkrankungssymptome ähnlich aber trotzdem andere als bei Mutationen im Fibrillin-Gen FBN1. Um die Krankheitsentwicklung besser zu verstehen, wurden bisher mehr als 560 unterschiedliche Mutationen im Fibrillin-Gen analysiert. Die Untersuchungen sind recht kompliziert, denn allein das Fibrillin-Gen FBN1 besteht aus 235 kb DNA und enthält 65 Exons. Das gesamte Gen ist Ziel der Mutationen, dennoch dominieren die Exons 26-28.

Bei Paganini wurde aufgrund der Überdehnbarkeit der Gelenke und der Haut, der dünnen und langen Finger sowie der Körperstatur, ohne den Patienten selbst zu kennen, auf ein Marfan-Syndrom geschlossen. Andere Erkrankungsmerkmale des MarfanSyndroms wurden bei ihm nicht dokumentiert und sind bisher auch nicht bekannt. Zum Marfan-Syndrom gehört allerdings ein vielfältiger Komplex von Symptomen, die unterschiedlich ausgeprägt sein können. Möglicherweise fehlten bei Paganini solche zusätzlichen Symptome oder sie wurden aufgrund des medizinischen Standards des frühen 19. Jahrhunderts von seinen Ärzten nicht erkannt. Neben Defekten in den Gelenken, den Knochen und in der Haut treten bei den Patienten noch funktionelle Defekte durch eine Lungenüberdehnbarkeit auf, außerdem wird 
durch strukturelle Schwächen am Herzklappenstützgerüst das gesamte kardio-vaskuläre System beeinflusst. Die Aorta kann sich ausweiten und dabei reißen, was meist zum Tod der Patienten führt. Daneben kommen bei den Erkrankten noch Defekte in den Hirnhäuten vor, und die Augenlinse kann nicht mehr exakt fixiert werden, was zu Sehstörungen führt.

Erkrankungsmerkmale eines Marfan-Syndroms können sich nicht nur durch den gestörten Aufbau der Mikrofibrillen in den Bindegeweben ergeben, sondern auch durch den Abbau des defekten Fibrillinproteins. Tatsächlich werden im Harn der Patienten vermehrt hydroxyprolinhaltige Peptide sowie Glykosaminoglykane ausgeschieden.

DNA-Proben konnten von Paganini noch nicht analysiert werden, sodass es keine gesicherte Hinweise auf ein defektes Gen gibt. Es ist unwahrscheinlich, dass eines Tages noch mögliches Untersuchungsmaterial auftaucht. Kopfhaare, die er als Andenken zahlreichen Verehrerinnen überließ, waren geschnitten und es fehlen Haarwurzeln, die heute eine Genanalyse erlauben könnten. Sein Sohn Achille, der nicht wie sein Vater erkrankt gewesen war, hatte allerdings Nachkommen. Zwei Ururenkel von Paganini haben sich inzwischen für eine Genanalyse zur Verfügung gestellt. Ein defektes Fibrillin-Gen wurde bei ihnen nicht gefunden. Die Leiden des Niccolo Paganini werden deshalb im Rückblick rätselhaft bleiben [6,7].

\section{Angeborene Defekte des Bindegewebes}

Das Marfan-Syndrom stellt die größte erbliche Gruppe von Bindegewebserkrankungen dar und kommt mit einer Häufigkeit von 1 zu 10000 vor. Zwischen Männern und Frauen ist der Defekt gleichmäßig verteilt und es gibt auch keine geografische Häufung. Andere angeborene Störungen des Bindegewebes bilden die Ehlers-Danlos-Syndrome, das Pseudoxanthoma elasticum, das Osteogenesis imperfecta sowie die Mukopolysaccharidosen.

Neben dem Marfan-Syndrom haben noch die Ehlers-DanlosSyndrome eine historische Bedeutung erreicht und wurden in der Bevölkerung beachtet. Manche betroffene Patienten traten bei öffentlichen Veranstaltungen oder bei Varietees auf und ließen die Besucher erschauern. Die bekannten „Gummimenschen“, die früher zu vielen Zirkusprogrammen gehörten, konnten zum Beispiel die Haut der Stirn bis über die Nase ziehen, und „Schlangenmenschen“ waren in der Lage, in winzige Kisten zu kriechen oder ihre Gliedmaßen in einem für normale Menschen nicht nachvollziehbaren Umfang zu verrenken. Es war für sie kein Problem, die Füße hinter dem eigenen Genick zu kreuzen und sich dann zu entspannen.

Bei den Ehlers-Danlos-Syndromen treten erblich bedingte Störungen in der Kollagensynthese und der Kollagenreifung auf. Die Kollagenfibrillen der Patienten bleiben dünn und weisen nur eine geringe Zugfestigkeit auf. Die Haut lässt sich deshalb bei ihnen in extrem großen Falten vom Körper wegziehen, kann dabei allerdings auch schnell verletzt werden ( $\bullet$ Abb.4) [6-8].

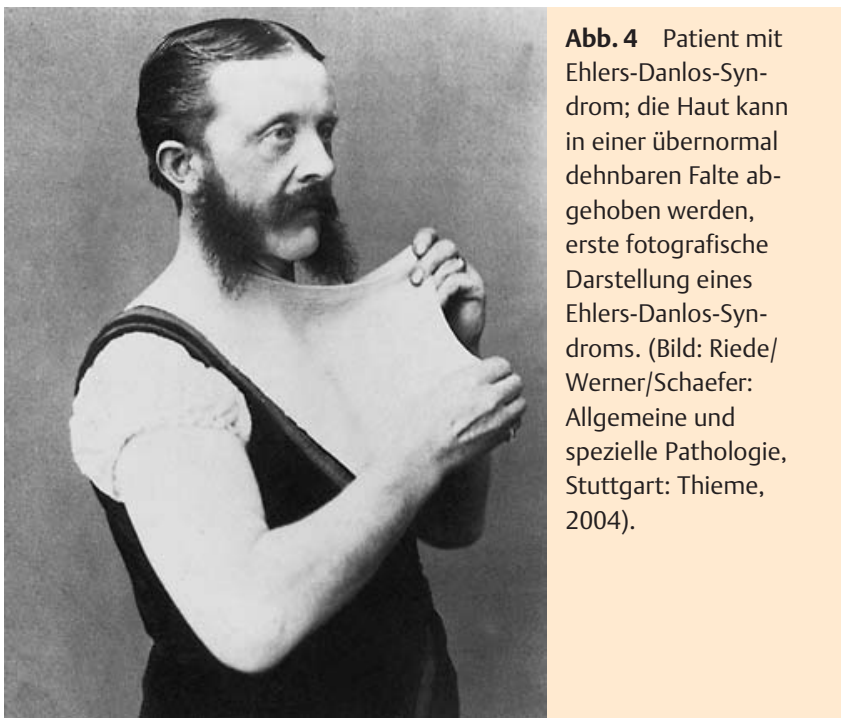

Abstract

\section{The Diseases of Niccolo Paganini}

The Italian violinist and composer Niccolo Paganini was wellknown in Europe because of his techniques playing the instrument. He developed techniques which no other violinist could reproduce. He was able to overstretch his joints in arms and fingers. Today, it is discussed that Paganini suffered from a marfansyndrome. This syndrome could allow him to develop his unique techniques. However, no diagnosis of the disease is possible in his descendants. Defects in fibrilline-genes were not detected in their genomes. No DNA-material is conserved from Paganini.

\section{Literatur}

1 Neill E. Niccolo Paganini. München: List, 1990

2 Kapp J. Niccolo Paganini. Tutzing: H. Schneider, 1989

3 Franken FH. Die Krankheiten großer Komponisten. Bd. 3: Niccolo Paganini, Richard Wagner, Georges Bizet, Gustav Mahler, Max Reger. Wilhelmshaven: Noetzel, 1991

4 Koldehoff N, KoldehoffS. Wem hat van Gogh sein Ohr geschenkt? Alles, was sie über Kunst nicht wissen. Frankfurt: Eichborn, 2007

5 Reitz M. Kunst und ärztliche Diagnose. In: Expedition in die Wissenschaft. Bd. 1. Weinheim: Wiley-VCH, 2006: 149-169

6 Graw J. Genetik. Heidelberg: Springer, 2006

7 Strachan T, Read AP. Molekulare Humangenetik. München: Elsevier Spektrum, 2005

8 Riede U-N, Werner M, Schäfer H-E (Hrsg). Allgemeine und spezielle Pathologie. Stuttgart: Thieme, 2004 